\title{
JULIÁN MARÍAS, JAIME BENÍTEZ Y LA FUNDACIÓN ROCKEFELLER
}

Servando Ortoll*

Annette Ramírez**

\section{Preámbulo}

$$
\text { Un dicho popular afirma que "más }
$$
vale llegar a tiempo que ser invitado". El desenlace es aún más feliz cuando el momento propicio coincide con la invitación hospitalaria. Ese fue el caso de la Universidad de Puerto Rico y los intelectuales españoles exiliados (forzosa o voluntariamente) durante el régimen de Francisco Franco. Siempre atento a atraer a la Universidad de Puerto Rico (UPR) estudiosos que proporcionaran lustre y prestigio a la institución, Jaime Benítez, rector del Recinto de Río Piedras desde 1942 y famoso por reclutar "a todo intelectual de mérito que se le ponía a tiro, y si tenía dificultades en su país, tanto mejor", ${ }^{1}$ incorporó a un nutrido grupo de artistas y profesores que dictaron cátedra, realizaron investigaciones, y asumieron el liderazgo en varios ámbitos de la vida intelectual en Puerto Rico. Los más reconocidos de este grupo fueron Pablo Casals y Juan Ramón Jiménez, pero muchos otros destacaron por sus aportaciones al conocimiento y a las artes en general, así como al quehacer universitario.

\footnotetext{
* Universidad de Sonora.

** Investigadora.

${ }^{1}$ Julián Marías, Una vida presente: memorias, 1989, Madrid, Alianza, 3 vols., 2: 125.
} 
Podemos mencionar, entre otros, a Eugenio Fernández Granell, Sebastián González García, Juan Marichal, Julián Marías, Alfredo Matilla y Pedro Salinas. La historia de cómo cada cual encontró un hogar en Puerto Rico es materia para múltiples relatos. En las páginas que siguen nos limitamos a narrar la experiencia del filósofo vallisoletano Julián Marías, vinculado con la UPR entre 1956 y 1961, años en que Jaime Benítez logró que la Fundación Rockefeller subvencionara a Marías para que, de manera periódica, visitara su universidad y escribiera una 'biografía intelectual' de su mentor y maestro, el recién finado filósofo madrileño, José Ortega y Gasset.

\section{Los primeros años}

Quizá la primera noticia formal que los funcionarios de la Fundación Rockefeller tuvieron sobre Ortega y Gasset -y su cercano colaborador Julián Marías- fue en 1950, cuando analizaron el discurso que el primero vertió dos años antes, al inaugurar un "Instituto de Humanidades" en Madrid. Los de la Rockefeller obtuvieron dicho discurso a través del rector y gran promotor educacional Jaime Benítez, quien escribió su tesis de doctorado para la Universidad de Chicago sobre Ortega y Gasset. Benítez admiraba al español "más que a nadie, lo había leído hasta saberse sus obras casi de memoria. Era un extraño discípulo [de Ortega y Gasset] a distancia, desconocido". ${ }^{2}$ Cuando Benítez supo que el filósofo español permanecería por un año en Aspen, Colorado, fue a visitarlo. Más tarde, lo acompañó en un viaje por Nueva York y Washington.

Como seguidor que era de Ortega y Gasset, Benítez, quien entonces apenas rebasaba los 40 años, buscó invitarlo por un año, sin éxito, a la Universidad de Puerto Rico. Ese fue el camino que transitó el discurso original de Ortega y Gasset. Al recibirlo de Benítez, los funcionarios de la Rockefeller se interesaron en el proyecto: ellos desarrollaban

\footnotetext{
${ }^{2}$ Ibid., 2: 123-4.
} 
entonces un programa de humanidades dentro de la misma Fundación. Les interesó en particular la parte del discurso sobre el Instituto de Humanidades apenas en ciernes, en la que Ortega y Gasset invitaba a los 'humanistas' españoles -en pleno régimen franquista- a que colaboraran en equipo:

Quisiéramos emprender una serie de estudios sobre las más diversas dimensiones en que se desparrama el enorme asunto 'vida humana'. Para ello buscamos una amplia colaboración. Desde hace mucho tiempo, en las ciencias naturales se trabaja en equipos. Las investigaciones sobre el hecho humano han llegado a un punto que reclama una organización parecida. El tamaño de lo que tal organización supondría... invita a la renuncia anticipada y a una inmediata parálisis. Por tanto, no se trata aquí de empresa semejante. Mas, ¿por qué no intentar un ejemplo y aun éste en formato minúsculo, de lo que podrían ser esos estudios y estas investigaciones en común? $?^{3}$

"Esta descripción [...] del Instituto en Madrid dirigido por Ortega y Gasset y Julián Marías”, escribió Chadbourne Gilpatric a sus colegas de la Rockefeller, "parece interesante y muestra una preocupación con algunos de los problemas que hemos discutido en conexión con nuestro programa". ${ }^{4}$ La coincidencia de perspectivas que Gilpatric encontró entre lo que proponían Ortega y Gasset y Marías y lo que la Fundación buscaba no cayó en el vacío, aunque tampoco rindió frutos inmediatos. No fue sino hasta seis años después que los de la Rockefeller recibieron noticias de Julián Marías, el colaborador más cercano de Ortega y Gasset desde 1932. Y las novedades vinieron por

${ }^{3}$ Pocantico Hills, North Tarrytown, Rockefeller Archive Center (en adelante RAC). Colección Rockefeller Foundation (en adelante RF). Record Group (en adelante RG) 2-1949. Series 789. Caja 467. Carpeta 3135. José Ortega y Gasset, "Sentido de las nuevas Humanidades". Anexo a carta de William Berrien a Chadbourne Gilpatric. Cambridge, Massachussets, 21 de julio de 1949.

${ }^{4}$ RAC. RF. RG 2-1950. Series: 789. Caja 505. Carpeta 3379. "The Aula Nueva Institute of Humanities". Correspondencia interdepartamental de Chadbourne Gilpatric a Charles B. Fahs, John Marshall y Edward F. D’Arms. Nueva York, 20 de marzo de 1950. 
conducto de Jaime Benítez, quien tras una visita de cortesía a Ortega y Gasset en Madrid, conoció en persona a Julián Marías y lo invitó a pasar una temporada en la Universidad de Puerto Rico.

\section{Estancia en Yale}

Dada su postura crítica al régimen de Franco, su cercanía con Ortega y Gasset y con el Instituto de Humanidades (que cofundó con Ortega y Gasset en 1948), y gracias, según insiste uno de sus hijos, a que un ex amigo de su padre lo delató frente a las autoridades españolas, Marías se desarrolló como filósofo al margen de la universidad en España. ${ }^{5}$ Para sobrevivir, aparte de recibir regalías por sus numerosas publicaciones, Marías estableció contactos con universidades norteamericanas: fue profesor visitante de Wesley College de 1951 a 1952; durante este último año ofreció un curso de verano en Harvard; en 1955 fue profesor invitado en la Universidad de California en Los Ángeles $\mathrm{y}$, un año más tarde, profesor visitante de filosofía en la escuela de postgrado de la Universidad de Yale. Entre 1952 y 1955 también fue profesor del bachillerato -el junior year-que el Smith College ofrecía todos los años en Madrid. ${ }^{6}$

De todas sus visitas a los colleges y universidades en Estados Unidos, la que más huella dejó en su persona, así como en la de sus estudiantes y colegas, fue su estancia en Yale, cuyo departamento de filosofía era considerado como el número uno de todas las universidades en Estados Unidos. Como parte de su contrato en Yale, Marías habría de dictar cursos, además de ofrecer, a su arribo, seis conferencias

${ }^{5}$ Véase Javier Marías, "El padre", en ídem., Vida del fantasma: entusiasmos, bromas, reminiscencias y cañones recortados, 1995, Madrid, El País/Aguilar, 425-8, en esp. 426.

${ }^{6}$ Consúltese el curriculum vitae de Julián Marías en RAC. RF. RG 1.2. Series: 243. Caja 10. Carpeta 68. Anexo a carta de Jaime Benítez a John Marshall, director asociado de las Humanidades en la Fundación Rockefeller. Río Piedras, Puerto Rico, 17 de julio de 1956. Es posible que Marías llamara 'bachillerato' a la licenciatura (Bachelor of Arts) estadounidense. 
públicas. ${ }^{7}$ En enero de 1956 Marías llegó a Nueva York, proveniente de Madrid, con su esposa Dolores -Lolita-Franco y sus cuatro hijos. De Nueva York se trasladaron a New Haven.

En Yale, además de tener 'excelentes' estudiantes, Marías se vio rodeado de estupendos colegas. En el departamento de filosofía de Yale -Marías lo recordó más adelante- se "estudiaba todo, las disciplinas filosóficas sin exclusión, y con insistencia en la metafísica; la historia de la filosofía de todos los períodos, sin la estrechez que sobrevino a muchos departamentos en años sucesivos". ${ }^{8}$

En Yale publicó Marías el primero de sus libros en inglés. Pero quizá lo más importante que logró fue desarrollar al máximo su capacidad de filosofar, equivalente, él lo confesó más tarde, a lo que había alcanzado en el Instituto de Humanidades en Madrid. No se encontraba Marías en casa pero mientras duró su estancia en Yale debió sentirse como tal: durante su residencia en la universidad llegó a tener, lo aseguró el tercero de sus hijos, 'pinta de americano'. ${ }^{9}$ Sus colegas filósofos consideraron a Marías un 'visitante inusual' dentro del departamento y le ofrecieron un puesto permanente de catedrático (full Professor) en el departamento de filosofía, pero Marías, ávido por regresar a España, rechazó la oferta.

La Universidad de Yale se portó conmigo con una cordialidad y estimación conmovedoras. Me ofreció un puesto permanente... en condiciones muy atractivas. Si no quería romper con los estudios españoles, podía dar, además de los cursos del departamento de Filosofía, uno en el de Español. Si aceptaba, lo tenía todo resuelto... Pero mi decisión de volver era inquebrantable. ${ }^{10}$

${ }^{7}$ Sobre su estancia en Yale, consúltese Julián Marías, Una vida presente, 2: 113-116.

${ }^{8}$ Julián Marías, Una vida presente, 2: 116.

9 Javier Marías, “Que por mí no quede’”, en ídem., Vida del fantasma: entusiasmos, bromas, reminiscencias y cañones recortados, 1995, Madrid, El País/Aguilar, 429-33, en esp. 429.

${ }^{10}$ Julián Marías, Una vida presente, 2: 119. 
Cuando se supo que Marías había resuelto marcharse de manera 'firme' e irrevocable, los miembros del Departamento de Filosofía, apesadumbrados pero respetuosos de su sentir, manifestaron por escrito lo que para ellos significó su paso por Yale y lo que simbolizaba su retorno a España. Correspondió al profesor Charles W. Hendel, el chairman del departamento - quien compartía con sus colegas su 'genuino interés personal' por el filósofo español- redactar una carta que resumía la presencia de Marías en el departamento y el sentir de sus colegas respecto al estudioso que había cambiado su forma de apreciar la filosofía. La carta, cuyo facsímil se encuentra en el Rockefeller Archive Center, señala en sus partes medulares:

No solicitamos que se uniera a nuestro departamento este año para tenerlo de ornato: nosotros lo pusimos a trabajar en un programa de seminarios muy lleno. Nunca habíamos tenido antes en nuestro cuerpo a un filósofo directo de España y queríamos que los juniors y seniors que estudiaban en nuestro departamento tuvieran la rara oportunidad de escuchar interpretaciones de filosofía y filósofos que nunca oirían de nosotros y de nuestra tradición particular...

Pero además de esos cursos le asignamos otros dos de postgrado, llamados "Imaginación y ficción”, y “Teoría de la vida humana". Aquí también usted trajo nuevos pensamientos y nuevas interpretaciones de figuras conocidas y evocó un interés y reflexión entusiastas en estos jóvenes eruditos que aspiran a la vocación de maestro y a la búsqueda de la filosofía. Sus enseñanzas han afectado el propio pensamiento de dichos jóvenes, como muchos de nosotros bien sabemos por sus repercusiones en nuestros propios seminarios. Dos filósofos en particular usted ha convertido en una presencia real y viviente para todos nuestros estudiantes y para nosotros: Husserl y, más profunda y conmovedoramente, Ortega. Sabemos que será una fuente duradera de gratificación para usted el percatarse lo que ha hecho aquí por la memoria de nuestro amado amigo y maestro. ${ }^{11}$

${ }^{11}$ RAC. RF. RG 1.2. Series 243. Caja 10. Carpeta 68. Carta de Charles W. Hendel a Julián Marías, fechada en Yale el 7 de junio de 1956 y reproducida en el Reporte Anual del Departamento de Filosofía en la Universidad de Yale. Anexo a extracto de carta de Charles 
Hendel concluyó su carta a Marías afirmando que sus colegas en el departamento lo consideraban 'uno de nosotros'. El sentimiento era mutuo: "Usted me ha repetido, también, cómo la experiencia de nuestras discusiones le recordaban los grandes días en Madrid, cuando los filósofos que diferían, resolvían sus diferencias en el espíritu de la filosofía verdadera. Usted se sintió, lo dijo, realmente en casa con nosotros." ${ }^{12}$ Pero Hendel sabía que Marías había resuelto partir -en España habría de editar la obra inédita de Ortega y Gasset, y dirigir el Instituto de Humanidades- y no quedaba más que desearle lo mejor:

Es en estos momentos de nuestra comprensión mutua de una comunidad genuina de interés y sentimiento que lo vemos marcharse para ocuparse de trabajos de piedad filosófica: la publicación de los últimos trabajos inéditos de Ortega y la conformación de un libro suyo en memoria de Ortega y finalmente la conducción del Instituto des [sic] Humanidades que ustedes establecieron. Hablamos ayer entre nosotros sobre los problemas que usted enfrenta para mantener viva la que posiblemente sea la única institución en la que la mente y el espíritu libres del hombre pueden alimentarse en su país... No podemos sino admirarlo por el valor y los sacrificios que usted hace para que haya erudición, filosofía y libertad en su tierra natal. Nuestros corazones están con usted mientras usted parte con tan finos intereses y resuelve emprender estas grandes cosas. ${ }^{13}$

"La carta -recordó tiempo después Marías-me conmovió profundamente, y sentí una profunda tristeza por dejar la Universidad de Yale y a los que habían llegado a ser mis amigos." 14 Marías, no resta duda, quedó transformado por Yale, por la facultad y por sus estudiantes. A su vez, los anfitriones de los Marías no se detuvieron en nada para mostrar su afecto y desconsuelo por su partida. La viuda del ex Presidente de la

W. Hendel a Edward F. D'Arms. Universidad de Yale. New Haven, Connecticut, 17 de septiembre de 1956.

\footnotetext{
${ }^{12}$ Ibid.

${ }^{13} \mathrm{Ibid}$.

${ }^{14}$ Julián Marías, Una vida presente, 2: 119.
} 
universidad, la señora Angell, 'una mujer de gran belleza y elegancia', "cuando íbamos a marcharnos, dio una espléndida recepción, seguida de cena para los más íntimos. Hendel le dijo [a la señora Angell]: 'Esta fiesta es para convencer a Marías de que se quede, ¿verdad?' [Ella le] contestó: 'No, ya sé que se va; es para que vuelva'". ${ }^{15}$

\section{Regreso a España, con escala en Puerto Rico}

Marías, hombre "con sombrero, gabardina o abrigo largos, ojos azules, el mentón partido y gafas redondas de concha", ${ }^{16}$ reconoció en esos momentos que partía por razones de 'piedad filosófica', pero atesoraba también motivos emocionales: consideraba su deber regresar a la Patria por su familia, y por no perder una presencia e influencia intelectual en España, que consideraba fundamental:

A pesar de todo, se fue decantando en mí la resolución de volver a España, de vivir en mi país, dentro de mi lengua, de continuar todas las cosas a las que había puesto mi vida. Estaba seguro de que Lolita, aunque los Estados Unidos le gustaban, no se sentiría feliz; en cuanto a mis hijos, entre ocho y dos años, si me quedaba serían sin duda americanos. Se dirá que esto era una ventaja; pero habían nacido españoles, y no me sentía autorizado a cambiar su destino. Además, pensaba que el ser español, con alguna calidad y esmero, no está nada mal. Finalmente, me gustaba mucho ser profesor de americanos, amigo de americanos; no me hubiera desagradado ser abuelo de americanos; pero ipadre de americanos me parecía demasiado! ${ }^{17}$

Dos eventos mostraron a Marías que, de permanecer en Yale, perdería el ascendiente intelectual que hasta entonces había mantenido sobre sus estudiantes estadounidenses, y el reconocimiento que había alcanzado como filósofo versado en la obra de Ortega y Gasset. El

\footnotetext{
${ }^{15}$ Ibid., 2: 121.

${ }^{16}$ Javier Marías, "Que por mí no quede”, 429.

${ }^{17}$ Julián Marías, Una vida presente, 2: 118.
} 
primero de dichos lances fue que la nueva coordinadora del junior year del Smith College, que se impartía en Madrid, preocupada por su propio bienestar político, y tras escuchar rumores de que Marías permanecería en Yale, lo "sustituyó por otro profesor, más grato al régimen español". ${ }^{18} \mathrm{Si}$ lo anterior mermaba los ingresos personales de Marías, un último evento lo conmovió más en lo personal y en lo profesional: los herederos de Ortega y Gasset le pidieron a otro filósofo que editara sus escritos desconocidos: "los hijos de Ortega pusieron en manos de Paulino Garagorri la preparación y edición de los manuscritos inéditos de su padre; Garagorri tenía indudable devoción por Ortega, pero sólo se había aproximado a él en el último año de su vida"; por lo visto, concluyó Marías, la familia de Ortega y Gasset no 'podía esperar unos meses' a su retorno. ${ }^{19}$

Este último hecho, tan injusto como inesperado -Marías no sólo era quien mejor conocía de la obra de Ortega y Gasset, sino que había sido su más cercano colaborador durante años-, lo llevó a madurar un plan que, de poseer los medios económicos para realizarlo, le permitiría salir bien librado de esta afrenta familiar: Marías concluyó que, en vez de recopilar y rescatar los trabajos inéditos e inacabados de su maestro, escribiría una biografía intelectual de Ortega y Gasset que desplegara su obra filosófica dentro del contexto más amplio de la sociedad europea y que describiera los tiempos que el filósofo español había vivido. Por lo pronto Marías debía volver a España, para rescatar el sitio que injustificablemente había perdido.

Aunque Marías sabía que, en Madrid, le "esperaban dificultades, riesgos, sinsabores y, lo peor de todo, decepciones", ${ }^{20}$ no titubeó en regresar. Antes de su retorno, sin embargo, Marías tenía obligaciones que cumplir. "Cuando Jaime Benítez supo que yo estaba en Yale",

${ }^{18}$ Ibid.

${ }^{19}$ Ibid.

${ }^{20}$ Ibid. 
recordó Marías, "me invitó a dar un cursillo breve al terminar los míos. Por eso aterricé un día en el aeropuerto de Isla Verde". ${ }^{21}$

Para Benítez el que Marías aceptara hacer escala en Puerto Rico, encarnó la oportunidad que buscaba para, una vez en persona, hacerle una propuesta que no pudiera rehusar. Cuando el rector Benítez le propuso que se afiliara al Recinto de Río Piedras, Marías ya tenía noticias sobre su persona: "su prestigio en los Estados Unidos era muy grande, y las grandes Fundaciones, Ford o Rockefeller, tomaban como evidente todo lo que les decía y proponía". ${ }^{22}$ En efecto, para los funcionarios de la Rockefeller resultaba difícil rechazar las propuestas de Benítez: aparte de ser originales, el rector las presentaba lleno de seguridad, persistencia, y entusiasmo.

\section{Marías y Ortega y Gasset}

Desde al menos 1950 Marías había proyectado componer un 'comentario perpetuo' a la primera obra (publicada en 1914) de su maestro y amigo José Ortega y Gasset, a razón de 'dos o tres líneas' por cada una del texto original. Para Marías, Las Meditaciones del Quijote contenían el núcleo del pensamiento orteguiano: de allí que a su arribo a Yale a principios de 1956 empezara a conformar dicha glosa para terminarla (posiblemente con más ánimos, dada la afrenta que había sufrido de la familia de Ortega y Gasset) en febrero de 1957, de regreso en Madrid. ${ }^{23}$ Marías le habló de su proyecto a Benítez, quien se "lo pidió para la Biblioteca de Cultura Básica de la Universidad de Puerto Rico. Fue un trabajo enorme y minucioso, que puso de relieve todo lo que había en ese libro, lo que significaba en su fecha, dentro del pensamiento filosófico". ${ }^{24}$

${ }^{21}$ Ibid., 2: 124.

${ }^{22}$ Ibid., 2: 125.

${ }^{23}$ Ibid., 2: 131.

${ }^{24}$ Ibid., 2: 131-132. 
Años más tarde Marías reconoció que, tras la muerte de su maestro (y el desaire que sufrió a manos de los herederos de Ortega y Gasset), su interés por él, por su obra y su figura, no había hecho más que aumentar.

Sabía además que Ortega contaba con mis interpretaciones [...], que estaba seguro de que 'nuestra' filosofía iba a continuar después de su muerte. Nada podía desanimarme de esa empresa. Apenas terminada la edición comentada de las Meditaciones, me dispuse a escribir el libro que Ortega merecía, el que yo le debía.

Era un enorme trabajo. Había que poner en claro, no sólo la situación en que España estaba cuando Ortega nació y actuó en ella, sino el pensamiento europeo en este siglo, muy particularmente el alemán. [...]

El trabajo que había hecho al comentar las Meditaciones había desbrozado el camino y me había mostrado qué se podía hacer. Pero era un trabajo largo, absorbente, de rendimiento lento y problemático. A Jaime Benítez se le ocurrió algo en lo que nunca hubiera pensado: pidió que la Rockefeller Foundation concediera un grant a la Universidad de Puerto Rico para que pudiese vivir mientras preparaba y escribía ese libro. ${ }^{25}$

No sorprende que a Benítez se le ocurriera cómo ayudar a Marías en sus propósitos. Este último anhelaba no sólo rendir un homenaje póstumo a su maestro Ortega y Gasset mediante una biografía intelectual que lo presentara frente al mundo occidental como la indiscutible autoridad filosófica que era, sino también colocarse ante el público conocedor, como el especialista único que era de la filosofía orteguiana. Este último anhelo se debió en parte a lo defraudado que Marías se sentía al no recibir el encargo, por parte de los herederos de Ortega y Gasset, de publicar los materiales inéditos de su maestro. Benítez ofreció auxiliar a Marías cuando percibió que éste se encontraba en la encrucijada de no poder dedicarse de manera exclusiva a su proyecto sin gozar de un apoyo económico suplementario: una vez de regreso

${ }^{25}$ Ibid., 2: 132. 
en Madrid, Marías tendría que buscar un empleo que sustituyera al que había perdido en el Smith College.

La propuesta de Benítez se resumía así: él obtendría fondos para que Marías se ocupara en Madrid de su proyecto sobre Ortega y Gasset; a cambio, Marías iría a Puerto Rico durante los veranos, a impartir unos cursos y poner al día a los estudiantes sobre los avances en su pesquisa. La propuesta le facilitaría a Marías elaborar su libro, a la vez que le permitiría revisar los materiales inéditos e inacabados de Ortega y Gasset, incluso aquellos en los que laboraba Paulino Garagorri. Benítez y Marías cerraron el trato. Sólo así Marías demostraría a los herederos de Ortega y Gasset el error en que habían incurrido cuando designaron a Garagorri -en vez de a él- para publicar los escritos inéditos de su padre. Faltaba ahora convencer a los de la Rockefeller de la relevancia del proyecto.

\section{Una propuesta para la Rockefeller}

El martes 17 de julio de 1956, Jaime Benítez dirigió una carta a John Marshall, director asociado de Humanidades de la Fundación Rockefeller. En ésta, Benítez delineó la propuesta que hizo a Marías. El rector de la Universidad de Puerto Rico presentó a Marías como maestro de la sesión veraniega en su universidad y como un filósofo en el cual estaba 'muy interesado'. Con algunos errores factuales (sabemos, por ejemplo, que Marías no publicaría los trabajos inéditos de Ortega y Gasset, y que tenía cuatro -y no tres- hijos), Benítez redactó cuidadosamente su carta para que Marshall le prestara cuidado y le ofreciera un respetable apoyo financiero adecuado a las necesidades del filósofo español:

Discípulo principal de Ortega y un brillante joven filósofo por méritos propios, Julián Marías desea dedicar los dos años próximos a preparar un libro sobre el gran maestro español. Marías está eminentemente capacitado para ello: trabajó con Ortega desde 1932 y los hijos de 
Ortega le han solicitado [sic] que revise y prepare para su publicación, los trabajos inacabados de su padre. Marías es un intelectual independiente en la España actual: ha decidido no enseñar en la Universidad de Madrid o trabajar en cualquier puesto oficial. Él depende de la venta de sus propios libros y artículos y de la tutoría privada para su sustento. Está casado y tiene tres hijos [sic]. Julián Marías es una especie de Mortimer Adler no beligerante, abiertamente católico, muy amable y poético. Lo que se rumora de que escribe a sus amigos en griego es falso, sólo en latín y eso en sus días estudiantiles. ${ }^{26}$

Añadió Benítez que Marías había regresado a Madrid tras “un muy exitoso contrato de enseñanza en Yale y un profesorado de verano en la Universidad de Puerto Rico". Después de subrayar que Marías era 'un profesor brillante', Benítez mencionó la oferta que le hizo el departamento de filosofía de Yale, indicando cómo el propio Benítez trató de "persuadirlo para que se quedara en la Universidad de Puerto Rico", y cómo el filósofo español había rehusado permanecer más tiempo en la isla, dado su compromiso por "preparar los trabajos inéditos de Ortega". ${ }^{27}$ Seguía ahora la parte delicada de su carta.

Me pregunto si hay alguna manera a través de la cual se le pudiera otorgar [a Marías] ayuda financiera para este importante trabajo en forma de una beca o subvención de algún tipo. Se me ocurre que 5,000 dólares, posiblemente renovados por un segundo año, serían adecuados. Esto se podría hacer ya a través de la Universidad de Puerto Rico o mediante una subvención directa. ${ }^{28}$

${ }^{26}$ RAC. RF. RG 1.2. Series: 243. Caja 10. Carpeta 68. Jaime Benítez a John Marshall director asociado de Las Humanidades, Fundación Rockefeller. Río Piedras, Puerto Rico, 17 de julio de 1956. La alusión a Mortimer Adler buscaba vincular al español con un distinguido estudioso estadounidense quien, hasta 1952, dictaba cátedra sobre filosofía del derecho en la Universidad de Chicago. Adler, junto con Robert Hutchins, se distinguió por publicar las "Grandes Obras" de la literatura y del pensamiento, proyecto del cual surgió un nuevo enfoque para el estudio de las humanidades en la Universidad de Chicago.

${ }^{27}$ Ibid.

${ }^{28}$ Ibid. 
Benítez adjuntó a su misiva dos documentos y la esperanza que la Fundación Rockefeller ayudara "al señor Julián Marías en este proyecto", ${ }^{29}$ que en parte era también de Jaime Benítez. Los anexos a su carta correspondían al impresionante curriculum del doctor Julián Marías, y a una propuesta que Marías redactó en inglés sobre el libro que escribiría en torno a José Ortega y Gasset, de recibir la beca o subvención de la Rockefeller. La propuesta, fechada el 29 de junio de 1956 en Río Piedras, consistía en cinco 'requisitos' fundamentales, el primero de los cuales leía así:

[El libro] debe mostrar el origen y crecimiento del pensamiento de Ortega y la génesis de su trabajo a partir de una situación doble: la vida española a vuelta de siglo y el trasfondo intelectual europeo de esos momentos. Las posibilidades y el significado del trabajo de Ortega, tanto social como filosófico, pueden ser entendidos sólo colocándolos frente a ese escenario dual.

Un libro con tales requerimientos, afirmaba Marías, no sería fácil de consumar, pues presuponía "un conocimiento completo y profundo de todos los escritos y cursos de Ortega, así como un amplio conocimiento de primera mano tanto de la situación histórico-social de España como del pensamiento europeo contemporáneo". ${ }^{30}$ En suma, nadie como él se encontraba capacitado para la labor que proponía. Para completar el libro se necesitaría, además, de "un esfuerzo para actualizar todos los escritos de Ortega, tanto publicados como inéditos, y sobre todo una mediación personal sobre su filosofía y el desarrollo de las posibilidades de su método". 31

Marías cerró su propuesta afirmando que un libro como el que esbozaba merecía el 'intento de escribirse'. Le preocupaba además que una gran parte de la obra de Ortega y Gasset se perdiera, dada la "estructura misma de sus escritos y las condiciones de su medio". Para

\footnotetext{
${ }^{29}$ Ibid.

${ }^{30}$ Ibid.

${ }^{31}$ Ibid.
} 
Marías el trabajo de Ortega representaba "la primera experiencia de filosofía total hecha por individuos hispanohablantes". Marías equiparó incluso el papel que desempeñó Ortega y Gasset para los hispanohablantes con lo que Descartes hizo para los franceses, Francis Bacon para los ingleses, y Leibniz y Kant para los alemanes: Ortega y Gasset, "por lo tanto, representa una de nuestras más grandes posibilidades históricas". ${ }^{32}$

Unos días después, y desde la Ciudad de México, Benítez retomó con los de la Rockefeller el caso Marías y su propuesta para el libro sobre Ortega y Gasset. Su tesón era la clave del éxito entre los funcionarios de la Fundación. "Marías - escribió Benítez con letra apresurada a John P. Harrison- fue el estudiante más destacado de Ortega y es un maestro brillante y filósofo por méritos propios. El año pasado le fue muy bien en Yale y en P.R. [...] Nunca escribo cuando viajo y muy rara vez mientras me encuentro en casa. Así que debe usted saber que le concedo gran importancia a este asunto." 33

\section{La Rockefeller responde}

Que la carta de Benítez impactó desde un principio lo atestigua el que Chadbourne Gilpatric, subordinado de Harrison dentro de la Fundación, se aprestara a responderla de inmediato. Gilpatric reconoció ante Héctor Estades, administrador universitario a quien Benítez asignó para que se encargara del asunto, que la propuesta de Benítez había creado 'una profunda impresión inicial', ya que, entre otras cosas, Marías estaba "singularmente equipado para llevarla a término". ${ }^{34}$

${ }^{32}$ RAC. RF. RG 1.2. Series: 243. Caja 10. Carpeta 68. Propuesta de libro anexa a carta de Jaime Benítez a John Marshall, director asociado de Las Humanidades, Fundación Rockefeller. Río Piedras, Puerto Rico, 17 de julio de 1956. Las cursivas aparecen como subrayados en el original.

${ }^{33}$ Ibid.

${ }^{34}$ RAC. RF. RG 1.2. Series: 243. Caja 10. Carpeta 68. Chadbourne Gilpatric al señor Héctor Estades. Nueva York, 27 de julio de 1956. 
Pero Gilpatric no ofreció apoyo alguno al proyecto por parte de la Fundación. "Preferimos", advirtió, "por razones que estoy seguro usted puede entender, conocer de primera mano a un académico, antes de considerar de manera definitiva una solicitud de auxilio para su trabajo". ${ }^{35}$ Había, además, una serie de preguntas a las que la propuesta no respondía. Entre otras, en el supuesto que Marías escribiera el libro, “¿dónde y quién lo publicaría y con qué seguridades apropiadas de antemano? ¿Aparecería en español o en inglés y cuáles serían las probabilidades para que hiciera una traducción temprana?". ${ }^{36}$

Los de la Rockefeller querían leer la propuesta con más detenimiento y buscar un 'parecer competente' antes de contestar de manera definitiva a la carta de Benítez. Requirieron dos meses para investigar más a fondo el tema y, sobre todo, la persona. Edward F. D'Arms, funcionario de la Rockefeller, telefoneó a un viejo conocido de Marías: Charles W. Hendel, del departamento de filosofía en Yale. Unos fragmentos de la conversación de ambos proveerá la tónica de su charla:

EFD [Edward F. D’Arms] le pidió a H [Hendel] su opinión sobre Julián Marías. H estaba muy entusiasmado con Marías como persona y como profesor visitante en Yale [...]. H considera a Marías el más destacado filósofo liberal español hoy en día. Fueron Ortega y Gasset y Marías quienes fundaron el Instituto de Humanidades en Madrid para mantener viva cierta llama de libertad. Marías retornó a España para continuar el trabajo del Instituto pese a la desconfianza con la que el gobierno lo considera. $\mathrm{H}$ agregó que a Marías se le aprecia mucho por todo Sudamérica. ${ }^{37}$

Restaba la pregunta clave: ¿qué pensaba Hendel del libro que Marías proponía?

${ }^{35}$ Ibid.

${ }^{36}$ Ibid.

${ }^{37}$ RAC. RF. RG 1.2. Series: 243. Caja 10. Carpeta 68. Entrevista telefónica de Edward F. D’Arms con Charles W. Hendel. ¿Nueva York/New Haven?, 17 de septiembre de 1956. 
En cuanto a la propuesta específica de que Marías escriba un libro sobre Ortega, $\mathrm{H}$ se mostró entusiasta y expresó la esperanza de que la RF [Fundación Rockefeller] pueda auxiliarlo. H siente que la asociación con la Universidad de Puerto Rico sería indudablemente beneficiosa para Marías al brindarle la oportunidad de trabajar libremente sin presión gubernamental. ${ }^{38}$

La entrevista telefónica de Edward F. D’Arms con Hendel rindió prontos beneficios. Hendel coincidió con las palabras elogiosas que Benítez pronunció sobre Marías: ambos concordaban en que Marías estaba preparado para realizar una obra de gran trascendencia sobre Ortega y Gasset; que necesitaba fondos para trabajar, y que la Rockefeller era la más indicada para proporcionárselos.

\section{La Fundación se acerca a Marías}

Tras su llamada con Hendel, Edward F. D’Arms se comunicó con Marías, a quien había conocido el año anterior. Aunque ahora D'Arms contaba con informes adicionales sobre la trayectoria profesional de Marías, requería de más detalles sobre su proyecto. Haciendo eco a lo que Gilpatric solicitó de Héctor Estades, D’Arms preguntó: ¿cuál sería el enfoque del libro?, ¿cuál sería su relevancia?, ¿a qué público iría dirigido? Había además otras preguntas que requerían respuestas antes de que la Rockefeller considerara la solicitud:

¿Cuánto tiempo estima que le tomaría escribir el libro que planea, y cuál sería la naturaleza y el monto del apoyo que usted necesitaría? ¿Se han hecho arreglos para que se publique? Presuntamente el libro se escribiría y publicaría en español, pero en el fondo parecería deseable que existiera una traducción inglesa de éste. ¿Han mostrado algunos editores norteamericanos o británicos, en sus conversaciones, interés en

${ }^{38}$ Ibid. 
este sentido? Por último, ¿dónde planearía usted realizar la mayor parte de la pesquisa y redacción? ${ }^{39}$

Los de la Rockefeller precisaban saber cuán vinculado estaba Marías con la Universidad de Puerto Rico. "Dado que la Universidad de Puerto Rico se ha interesado en su proyecto, hemos inferido que usted podría pasar al menos parte de su tiempo allí, pero nos encantaría recibir más detalles sobre sus planes", ${ }^{40}$ escribió D’Arms.

A los cuatro días de redactada la carta de D'Arms, Marías respondió desde Madrid. Le complacía que la Fundación considerara sus planes para un libro sobre Ortega y Gasset. Según Marías, el rector de la Universidad de Puerto Rico coincidía con que su libro contribuiría tanto a comprender como a difundir "una filosofía cuyas posibilidades no han sido suficientemente desarrolladas". ${ }^{41}$ Su libro, aclaró Marías, no sería una biografía, sino "una interpretación filosófica del trabajo de Ortega" que incluiría "un elemento biográfico, dado el carácter dramático de los escritos de Ortega, es decir, la referencia a una situación personal e histórica, lo cual es uno de los elementos esenciales de la filosofía, según su propia visión". ${ }^{42}$ Por otra parte, sería difícil exponer su pensamiento de manera efectiva, sin que se intentara "reconstruir las líneas principales de su verdadera y altamente sistemática filosofía”.

Mi plan es tomar gran parte de las ideas de Ortega para mostrar cómo provienen de una intuición fundamental, una forma de entender la realidad, que se encuentra conectada de cerca a un método de investigación que puede, si se desarrolla adecuadamente, ser de gran ayuda en todos los campos de las humanidades... Siento que estoy en la posición de hacer comprensible el significado y amplitud de una doctrina que,

${ }^{39}$ RAC. RF. RG 1.2. Series: 243. Caja 10. Carpeta 68. Edward F. D’Arms a Julián Marías. Nueva York, 19 de septiembre de 1956.

${ }^{40}$ Ibid.

${ }^{41}$ RAC. RF. RG 1.2. Series: 243. Caja 10. Carpeta 68. Julián Marías a Edward F. D’Arms. Madrid, 23 de septiembre de 1956.

${ }^{42}$ Ibid. La palabra en cursivas aparece subrayada en el original. 
pese al hecho de que Ortega era y es ampliamente leído y comentado, permanece casi desconocido en segmentos muy grandes. ${ }^{43}$

Aunque 'primordialmente' se orientaría a un público interesado en filosofía, su libro, según Marías, interesaría a 'un publico más amplio', al menos en España y en América Latina, en donde la influencia de Ortega y Gasset "ha sido extremadamente profunda en todos los campos y todavía está creciendo". No habría dificultad alguna en publicar el libro "ya sea en España o en Argentina". Además, tanto Hendel como el señor Eugene Davidson, editor responsable de la Yale University Press, conocían sus planes de escribir el libro y se interesaban por publicarlo en inglés. ${ }^{44}$

A Marías le atraía emprender el estudio sobre Ortega y Gasset: si rechazó un nuevo nombramiento en Yale y desdeñó invitaciones de otras universidades para el año siguiente, era porque "quería trabajar sobre Ortega y tenía que pasar un tiempo en España”. Pero había una dificultad:

el único problema es que... no tengo un puesto oficial aquí y mi familia es bastante numerosa: mi esposa y cuatro niños, y sin ninguna ayuda financiera, yo tendría que iniciar otro trabajo, y esto retrasaría la escritura y publicación del libro. Si me pudiera dedicar enteramente a él, pienso que el libro estaría terminado en cerca de dos años, es decir, en 1958; si escribo un libro en dos volúmenes el primero estará ciertamente publicado antes de junio de $1958 .^{45}$

Marías anticipaba trabajar en gran medida desde Madrid. Había, sin embargo, discutido con el rector Benítez sobre "la posibilidad de impartir un curso o una serie de conferencias en la Universidad de Puerto Rico, que significarían una anticipación en grandes porciones del contenido del libro, antes de que se publicara. Sería posible hacer

${ }^{43}$ RAC. RF. RG 1.2. Series: 243. Caja 10. Carpeta 68. Julián Marías a Edward F. D’Arms. Madrid, 23 de septiembre de 1956.

${ }^{4}$ Ibid.

${ }^{45}$ Ibid. La palabra en cursivas aparece subrayada en el original. 
lo mismo", agregó Marías mostrando que se inclinaba por retornar al continente americano, "en alguna universidad en Estados Unidos". ${ }^{4}$

Marías sugirió a D’Arms que sus planes para reorganizar el Instituto de Humanidades en Madrid dependerían en gran medida de que la Rockefeller lo ayudara a publicar su libro. ${ }^{47}$ Entre líneas podía leerse que el patrocinio norteamericano le permitiría vivir con holgura para dedicarse tanto a su libro como a reestructurar el Instituto de Humanidades. Pero los funcionarios de la Rockefeller, en ese momento al menos, no se dieron por enterados.

\section{Benítez vuelve a la carga}

En un viaje a Nueva York en septiembre de 1956, Benítez visitó las oficinas de la Rockefeller. Allí habló con D’Arms en torno al proyecto de Julián Marías. El libro propuesto sería, lo afirmó Benítez, “el intento más importante para dar coherencia explícita a la filosofía de Ortega" y con seguridad lo publicaría la Revista de Occidente, dado que había dado a la estampa gran parte de la obra de Ortega y Gasset y que también se encargaba de editar los clásicos españoles de la Universidad de Puerto Rico. ${ }^{48}$ En lo que sigue aparece el proyecto de Benítez, que logró 'vender' con facilidad al funcionario de la Rockefeller:

B [Benítez] propone nombrar Marías profesor investigador en la Universidad de Puerto Rico y hacer que instruya durante el verano en torno a lo que investigó sobre Ortega el año precedente. El resto del tiempo Marías estaría libre para dedicarse a su propia investigación gran parte de la cual, presuntamente, la realizará en España. La Universidad de Puerto Rico estaría dispuesta a pagar el salario de Marías por el curso de verano y a administrar una subvención por el total del proyecto. B siente que 5,000 dólares anuales bastarían para atender las necesidades de Marías,

${ }^{46} \mathrm{Ibid}$.

${ }^{47}$ Ibid.

${ }^{48}$ Véase RAC. RF. RG 1.2. Series: 243. caja 10. Carpeta 68. Entrevista de Edward F. D’Arms con Jaime Benítez. Nueva York, 28 de septiembre de 1956. 
pero además requeriría el viaje de ida y vuelta de España a Puerto Rico. EFD [Edward F. D'Arms] le habló a B sobre la carta reciente de Marías y se acordó que EFD le escribirá a Marías para saber si necesitaría dos o tres años y a exhortarlo a que se ocupara de los detalles con B. B está preparado para actuar rápidamente y le solicitó a EFD que le enviara una copia de su carta a Marías. ${ }^{49}$

D’Arms concluyó que Marías requeriría, además de lo que la Universidad de Puerto Rico habría de proveer, "fondos para permitirle llevar a cabo su pesquisa por un período aproximado de tres años, además de viajes de ida y vuelta de España a Puerto Rico por dos o tres veranos". ${ }^{50}$ Luego de la visita de Benítez, D’Arms redactó una carta para Marías, solicitándole que a su vez le escribiera a Benítez para discutir los detalles del plan.

Marías se apresuró a contestarle a D’Arms informándole sobre sus propósitos: "Me complacerá dictar una cátedra en la Universidad de Puerto Rico durante los dos o tres próximos trimestres de verano." 51 Según Marías, “el período de tres años para mi pesquisa y escritura es por supuesto un máximo. Espero que el libro esté terminado y listo para publicarse antes de que termine este período". ${ }^{52} \mathrm{Su}$ certidumbre de que concluiría el libro antes de la fecha límite descartaba las contingencias que se le podrían presentar a Marías en los próximos 36 meses. En su carta, Marías señaló que al menos tres editoriales -la Revista de Occidente, administrada por el hijo de Ortega y Gasset; la Biblioteca de Cultura Básica de la Universidad de Puerto Rico, y Emecé Editores de Buenos Aires- se interesarían por publicar su libro sobre Ortega y Gasset. ${ }^{53}$

${ }^{49}$ Ibid.

${ }^{50}$ RAC. RF. RG 1.2. Series: 243. Caja 10. Carpeta 68. Carta de Edward D’Arms a Julián Marías, con copia para el doctor Jaime Benítez. Nueva York, 4 de octubre de 1956.

${ }^{51}$ RAC. RF. RG 1.2. Series: 243. Caja 10. Carpeta 68. Julián Marías a Edward F. D’Arms. Madrid, 6 de octubre de 1956.

${ }^{52}$ Ibid. La palabra en cursivas está subrayada en el original.

${ }^{53}$ Ibid. 
Benítez, mientras tanto, intervino de nuevo. Puesto que D'Arms y Marías estaban de acuerdo, no quedaba sino reformular la solicitud de subvención a la Rockefeller, que resumiría todos los pasos tornados hasta ese día, con un añadido que no aparece en otra parte: la petición de que, al término de su pesquisa, Marías pasara en Puerto Rico al menos un año académico:

A nombre de la Universidad de Puerto Rico solicito una subvención de $\$ 17,000$ dólares para ser utilizados durante un período de tres años en un proyecto que prevé un estudio importante sobre la obra del finado José Ortega y Gasset, su importancia en el mundo de habla española y sus repercusiones en la filosofía y cultura modernas. [...] estoy persuadido que tal proyecto contribuirá significativamente al campo de estudios españoles y confío que Julián Marías es eminentemente capaz de llevarlo a término. A la Universidad de Puerto Rico le interesa profundamente patrocinar este proyecto y está preparada para contratar a Julián Marías, como profesor universitario, para llevarlo a cabo. Este proyecto tomaría de dos a tres años y requeriría que Marías pase la mayor parte de su tiempo en España, estudiando los muy abundantes escritos inéditos de Ortega y Gasset y utilizando los vastos archivos de su familia, a los que ya tiene acceso, como asistente principal y pupilo de Ortega. Sin embargo, esperaríamos que Julián Marías venga a Puerto Rico periódicamente cada nueve meses o antes, para propósitos de consulta y enseñanza. Contaríamos además con que tras terminar y publicar su pesquisa, el profesor Julián Marías vendría a la Universidad de Puerto Rico por al menos un año académico, con un salario no menor que el que paga la Universidad de Puerto Rico a sus profesores regulares en dedicación exclusiva. ${ }^{54}$

La Universidad de Puerto Rico pagaría al doctor Julián Marías, de 'sus propios fondos', según Benítez, los intervalos de enseñanza que mencionó en su propuesta, así como por "el año de cátedra que siga a la publicación de su trabajo". La universidad también se aseguraría

${ }^{54}$ RF. RG 1.2. Series: 243. Caja 10. Carpeta 68. Carta de Jaime Benítez al doctor Edward F. D'Arms. Piedras Negras, 11 de octubre de 1956. 
de que se publicara el libro de Marías, "ya sea a través de salidas de mercados comerciales o como una publicación universitaria". ${ }^{55}$ En suma y de aceptarse la propuesta, durante tres años Marías recibiría \$5,000 dólares por los nueve meses que pasara en España, más \$2,000 dólares adicionales que cubrirían sus gastos de transporte entre Madrid y San Juan. ${ }^{56}$

\section{La Rockefeller se resuelve}

El martes 29 de enero de 1957, la Junta de Gobierno de la Fundación Rockefeller acordó subvencionar a Julián Marías con una cantidad $\$ 17,000$ dólares, que recibiría a través de la Universidad de Puerto Rico a lo largo de 36 meses. Las gestiones de Jaime Benítez habían triunfado. Durante los siguientes tres años -tiempo suficiente para terminar el libro (o libros) que Marías se había comprometido escribir- el filósofo español estaría exento de presiones financieras. El dictamen de la Rockefeller que otorgaba la subvención a Marías repetía los argumentos de Benítez, es decir, que el proyecto prepararía "la primera versión completa del sistema filosófico del español Ortega y Gasset" y que Julián Marías era idóneo para consumar el proyecto.

Julián Marías está extraordinariamente capacitado para llevar a cabo este proyecto. Marías fue el cofundador, con Ortega, del Instituto de Humanidades en Madrid, una institución carente de fondos para la investigación, por no estar vinculada con el gobierno español; Marías fue un estudiante y colega cercano de Ortega por casi un cuarto de siglo; tiene un amplio conocimiento de primera mano del entorno histórico y social del pensamiento español y europeo durante la época en que Ortega escribió, y tiene acceso completo a los archivos de la familia Ortega [...]. La capacidad profesional de Marías queda validada por sus publicaciones

\footnotetext{
${ }^{55}$ Ibid.

${ }^{56}$ Ibid.
} 
y por sus asociados en Yale, la Universidad de California en Los Ángeles y la Universidad de Puerto Rico, en donde ha impartido cátedra. ${ }^{57}$

En la resolución para otorgarle la beca, se afirma que el monto total de \$17,000 dólares sostendría a Marías en España durante 'tres períodos de nueve meses cada uno', y que dicha cantidad cubriría sus gastos de viaje a Puerto Rico, “donde espera impartir cátedra dos trimestres de verano durante el período que se extienda la subvención y por el mínimo de un año académico después de que termine el subsidio. Su salario, mientras imparte cursos, lo pagará la Universidad de Puerto Rico". ${ }^{58}$ La Universidad de Puerto Rico actuaría así como "una plataforma desde la cual el profesor Marías establecería contactos directos con estudiantes, instructores y figuras profesionales de casi todos los países" de América Latina, y como un "punto de enlace intelectual único" en el continente americano. ${ }^{59}$

\section{Marías, becario de la Rockefeller}

Una vez que la Rockefeller envió los fondos de la subvención a la Universidad de Puerto Rico, Benítez y Marías acordaron que éste podría ir a la isla entre junio y julio de ese año o esperar hasta enero o abril de 1958. Pero terminar su obra magna sobre Ortega y Gasset y visitar Puerto Rico durante los veranos para informar a sus futuros

${ }^{57}$ RAC. RF. RG 1.2. Series: 243. Caja 10. Carpeta 68. Resolución de beca para Julián Marías (RF 57024 PHILOSOPHY). Nueva York, 29 de enero de 1957.

${ }^{58} \mathrm{Ibid}$.

${ }^{59}$ Ibid. La Universidad de Puerto Rico, en esos apasionantes años, era lugar de encuentro no sólo entre latinoamericanos sino entre el primer y el tercer mundo: entre intelectuales anglosajones y sudamericanos. Esto se veía particularmente en los congresos que organizaba Benítez y en los que participaban funcionarios de la Rockefeller, como el mismo John P. Harrison quien servía de intermediario entre personas tales como la dramaturgo estadounidense Lillian Hellman y el escritor mexicano Jaime García Terrés. Véase Jaime García Terrés, "Lillian Hellman", en idem, El teatro de los acontecimientos, 1988, México, El Colegio Nacional/Ediciones Era, 11-13, en esp. 11. 
estudiantes sobre los adelantos de su trabajo, eran dos de los muchos proyectos por los cuales Marías se interesaba.

Liberado de ataduras financieras, Marías se dedicó a luchar en varios frentes que no necesariamente eran compatibles o complementarios: a dar a conocer mejor a Ortega y Gasset en el extranjero; a realizar publicaciones diversas; a fortalecer el Instituto de Humanidades en Madrid y a informar -también en el extranjero- lo que en España ocurría a los miembros de dicho Instituto y, en importante medida, a criticar el régimen de Franco desde dentro y desde fuera de España, dentro y fuera del Instituto de Humanidades. Por importante que fuera su labor multifacética -Marías parecía irrefrenable ahora-había un problema de fondo bastante serio: la Rockefeller le había otorgado la subvención para la primera de sus tareas; las demás se relacionaban con cuestiones políticas, más allá de las preferencias personales -e institucionales-de los miembros de la Fundación.

En cuanto a su meta de que se conociera más a Ortega y Gasset en el extranjero, Marías publicó un primer trabajo sobre Ortega y Gasset en su edición comentada de las Meditaciones del Quijote. Marías también obtuvo un ejemplar para la Rockefeller de El Hombre y la Gente -el primer volumen de la obra póstuma de Ortega y Gasset- que la Revista de Occidente (prueba de que para entonces tenía cierto ascendiente sobre los hijos del filósofo) imprimió especialmente para la Fundación. ${ }^{60}$ Que para Marías resultaba trascendental informar fuera de su país sobre el estado en el que se hallaban las humanidades y los humanistas españoles, puede verse en una de sus cartas a la Fundación Rockefeller:

Estoy cada vez más preocupado acerca de las posibilidades de la investigación independiente en el campo de las humanidades. Hace cuatro o cinco meses, una veintena de académicos y autores destacados [...] solicitaron permiso para una revista académica mensual, dedicada al estudio de filosofía, sociología, historia, literatura, filología y las

${ }^{60}$ RAC. RF. RG 1.2. Series: 243. Caja 10. Carpeta 68. Julián Marías a John P. Harrison. Madrid, 5 de octubre de 1957. 
ciencias naturales. No pudimos obtener este permiso y la revista no será publicada. Por otra parte, el público está cada vez más ansioso por tener libros, artículos, conferencias o cursos por parte de académicos honestos e independientes. Durante los meses de febrero y marzo, un curso de conferencias sobre problemas sociales organizado por los estudiantes de la Facultad de Leyes de la Universidad de Madrid tuvo un éxito tremendo, con auditorios entusiastas de muchos cientos de estudiantes. Alrededor de 1,500 asistieron a la conferencia que impartí allí sobre 'Sociedad y clases sociales'. Si fuera posible organizar alguna actividad [permanente], pienso que la situación se mejoraría sustancialmente en uno o dos años. El problema es que ninguna actividad aislada, aunque fuera lo más exitosa, permite depender en ella para cualquier acción futura, y es muy difícil dar a las generaciones jóvenes la orientación que ellos tanto echan de menos. ${ }^{61}$

Deseoso de conseguir apoyo para el Instituto de Humanidades en Madrid, Marías visitó la sede de la Fundación Rockefeller en dos ocasiones durante el mes de junio de 1958. Lo primero que hizo en la Rockefeller fue hablar sobre su pesquisa, para dejar en claro que, "en general, su trabajo procede exactamente como se planeaba". ${ }^{62}$ A una pregunta que John P. Harrison le hizo sobre la calidad de los cursos filosóficos en la Universidad de Puerto Rico, Marías contestó que no "eran muy avanzados, pero que tenía la impresión que la calidad estaba haciéndose sentir lentamente". ${ }^{63}$ Afirmó además que su primer verano lo había "dedicado a una serie de conferencias a las que asistieron miembros de la facultad y gente de la ciudad, pero que este año estaba impartiendo un curso por crédito, y un seminario para cinco o seis estudiantes avanzados, que trabajarían sobre las innovaciones conceptuales en el pensamiento de Ortega". ${ }^{64}$

${ }^{61}$ RAC. RF. RG 1.2 Series 243. Caja 10. Carpeta 68. Julián Marías a John P. Harrison. Nueva York, 21 de marzo de 1958.

${ }^{62}$ RAC. RF. RG 1.2. Series: 243. Caja 10. Carpeta 68. Entrevista de John P. Harrison con el profesor Julián Marías. Nueva York, 9 de junio de 1958/11 de junio de 1958 (conversación continuada).

${ }^{63}$ Ibid.

${ }^{64}$ Ibid. 
Haciendo a un lado la discusión sobre cuestiones profesionales, Marías desvió su conversación con Harrison hacia lo político:

A Marías le preocupa profundamente la situación de los intelectuales en España, y particularmente la de aquellos que trabajan en las humanidades. Él insistió en la importancia de la censura, y en el impacto que tenía sobre todo lo que se hacía - desde publicar una revista o un libro, hasta el permiso para dar conferencias y cursos-. Señaló que los censores nunca dicen en realidad que algo no se puede realizar. Simplemente no dan su aprobación, subrayando -si se les pregunta- que el asunto está bajo consideración. Un resultado de esto, según Marías, es que los autores que quieren que se publiquen sus libros censuran sus propias obras tanto que su pensamiento cuando mucho es parcial, y en realidad ellos son más estrictos [consigo mismos] que los propios censores. M sintió que un posible apoyo de la RF a un programa de investigación sería de lo más importante, porque los grupos que ejercen la censura sentirían que tienen que dar una respuesta a una solicitud, y en este caso si la rechazaban, tendrían que proporcionar sus razones -y dado que esto sería embarazoso- seguramente que accederían a cualquier programa de estudio o curso razonable. ${ }^{65}$

Mientras se vendían libros con más rapidez, la situación política en España, de acuerdo con Marías, estaba 'deteriorándose'. Luego añadió un elemento que sintió podía importar a Harrison, su interlocutor: según Marías, tradicionalmente los intelectuales estaban a favor de Estados Unidos, pero en los últimos dos años esto había cambiado debido, en parte, a que los intelectuales españoles estaban fortaleciendo relaciones con países de Europa Occidental, en particular con Francia. Pero lo anterior también se debía al apoyo que Estados Unidos brindaba al gobierno de Franco y más particularmente a intelectuales que no eran respetados en el ámbito nacional. Preocupaba a Marías que, una vez que Franco cayera, no existiera un grupo respetado de intelectuales que pudiera dirigir el pensamiento de los españoles.

${ }^{65}$ Ibid. 
El único grupo capaz de tomar el poder intelectual y políticamente sería el de los comunistas, ya que constituyen el único grupo políticamente activo que trabaja clandestinamente en España, hoy en día. Marías esperaba que hubiera alguna forma para que la RF apoyara, tanto cuanto por su autoridad como por su dinero, una revista o un proyecto de investigación de equipo que le diera coherencia a un grande y disperso conjunto de intelectuales y les brindara un vehículo para mantener una estatura intelectual de manera tal que tengan una posición de autoridad frente al público cuando llegue el colapso final del gobierno de Franco. La otra sola alternativa, como $\mathrm{M}$ lo ve, es abandonar el campo de autoridad intelectual a los comunistas. ${ }^{66}$

Para no dejar todo en manos de los comunistas españoles, Marías deseaba saber si, en caso de que se creara una asociación de intelectuales en España con 'existencia corporativa y con el propósito de conducir investigación, dar conferencias y publicar una revista’, ¿recibiría dicha agrupación apoyo de la Fundación Rockefeller? El tipo de revista que Marías concebía se parecería a (y competiría con) la Revista de Occidente y el tipo de pesquisa en equipo que proyectaba era "la estructura social de España en la primera mitad del siglo diecinueve", "algo que sería significativo por sí mismo y no por su naturaleza imposible de ser aceptado por el gobierno de Franco, y que sería lo suficiente amplio para congregar a gente de varios intereses". ${ }^{67}$

Para Marías era crucial constituir tal grupo, puesto que instruiría a jóvenes ansiosos de aprender. "Tal asociación tendría, entonces, como uno de sus objetivos, el entrenamiento de un grupo de jóvenes intelectuales que, $\mathrm{M}$ siente, no tendrían otro lugar a dónde ir sino con los comunistas, para prepararse en lo académico y su formación intelectual, dado que la premisa primordial de estos jóvenes es su rechazo a la filosofía social y política del régimen actual". ${ }^{68}$ Aunque Harrison, funcionario importante de la Fundación, dedicó dos días a

${ }^{66}$ Ibid.
${ }^{67}$ Ibid.
${ }^{68}$ Ibid.. 
entrevistarse con Marías, su silencio respecto a lo que pensó de sus entrevistas con el filósofo es elocuente. ¿Buscaba Marías colocarse al frente de un grupo de intelectuales españoles con el apoyo de la Rockefeller? Harrison tomó notas de todo lo que Marías dijo pero no respondió a sus solicitudes.

\section{Los tiempos de la subvención}

Mientras contaba con el apoyo económico de la Rockefeller, Marías se ocupaba de impartir conferencias en diversos países latinoamericanos, sin que le dedicara el tiempo necesario a su libro sobre Ortega y Gasset. Amediados de octubre de 1958, Marías anunció que el primer volumen de su obra sobre Ortega y Gasset aparecería unos nueves meses después de lo acordado. ${ }^{69}$ Mientras tanto, las obras editadas de Ortega y Gasset competían con la suya: "el libro más importante de Ortega", reconoció Marías, "La idea de principio en Leibniz y la evolución de la teoría deductiva y un librito suyo sobre La idea del teatro serán publicados estos días. Como autor, [Ortega] está muy vivo". ${ }^{70}$ El tiempo seguía su paso. Hacia finales de enero de 1959, el libro de Marías aún no había aparecido y sus cursos en Puerto Rico se alejaban más de su pesquisa sobre Ortega y Gasset y se acercaban más a su propio trabajo. Para su 'siguiente curso en Puerto Rico', Marías explicó a Harrison:

Planeo dar un curso de conferencias sobre "La metafísica de la razón vital en el marco de la filosofía europea del siglo veinte", y conducir un seminario sobre los "Métodos de investigación en las estructuras sociales". Pienso que esto dará tanto a los estudiantes como a los instructores jóvenes un conocimiento cercano con las obras de Ortega publicadas

${ }^{69}$ RAC. RF. RG 1.2. Series 243. Caja 10. Carpeta 68. Julián Marías a John P. Harrison. 13 de octubre de 1958.

${ }^{70}$ Ibid. Los títulos en cursivas aparecen subrayados en el original. 
póstumamente, así como con algunos de mis escritos. Más aún, será de ayuda para investigaciones posteriores en la isla. ${ }^{71}$

Julián Marías se había convertido en Julián Marías y paulatinamente se alejaba del Marías intérprete de Ortega y Gasset, cuyo fantasma había llevado a cuestas desde su muerte cuatro años antes. Ahora que Marías trabajaba más en lo suyo y buscaba que en América Latina y Estados Unidos se reconociera su desarrollo filosófico, descuidaba sus obligaciones con la Rockefeller, si bien continuaba sus contactos con la Universidad de Puerto Rico y con el propio Jaime Benítez.

Mientras esperaba el libro prometido, es decir, desde finales de 1958 hasta principios de 1959, Harrison se comunicó con académicos españoles residentes en Estados Unidos para confirmar cuanto Marías le había dicho por escrito y en persona. Al final, por razones que desconocemos, la Fundación no apoyó el proyecto de Marías en España. Los documentos sugieren que hubo un silencio de aproximadamente un año en la correspondencia cruzada entre Harrison y Marías en torno a este frustrado proyecto del filósofo español. ${ }^{72}$

\section{El fruto de sus labores}

En abril de 1960, Julián Marías avisó a Harrison que su obra Ortega I: circunstancia y vocación, estaba impresa. El libro, de 570 páginas, contenía cerca de 500 de texto y 70 de notas al calce. "Aunque el estudio [sobre Ortega y Gasset] será completado (trabajo desde enero en el segundo volumen), éste es un libro independiente." 73

Tres meses después, desde Puerto Rico, Marías informó sobre el progreso de su ambicioso trabajo, el cual aumentaba en complejidad y extensión:

${ }^{71}$ RAC. RF. RG 1.2. Series 243. Caja 10. Carpeta 68. Julián Marías a John P. Harrison. Madrid, 26 de enero de 1959.

${ }^{72}$ Consúltese RAC. RF. RG 1.2 Series 243. Caja 10. Carpeta 68.

${ }^{73}$ RAC. RF. RG 1.2. Series 243. Caja 10. Carpeta 68. Carta de Julián Marías a John P. Harrison. Madrid, 21 de abril de 1960. 
He estado trabajando en el segundo volumen -y mirando el tercero-. Hay un largo camino por delante, pero espero que el resultado valga la pena. En cuanto regrese a España, seguiré escribiendo y pienso caminar deprisa, aunque demasiadas preguntas necesitan una clarificación a fondo. Usted puede ver en el primer volumen que el pensamiento de Ortega y sus implicaciones históricas - tanto españolas como europeas-se asemejan a un bosque tropical. ${ }^{74}$

El plazo de la subvención original, para entonces, había llegado a su fin. En agosto de 1960, Jaime Benítez informó el uso que la Universidad de Puerto Rico había dado a los $\$ 17,000$ dólares que la Rockefeller otorgó por un período de tres años - del 1 de marzo de 1957, a julio de 1960- para "la pesquisa y publicación por Julián Marías de un libro sobre el sistema filosófico de José Ortega y Gasset”. Julián Marías recibió $\$ 14,801.40$ dólares y se utilizaron $\$ 2,198.68$ dólares para sufragar sus gastos de viaje entre España y Puerto Rico en tres ocasiones. Aparte, la Universidad le pagó al profesor Marías, "de sus propios fondos" el trabajo que realizó en Puerto Rico durante esas tres estancias. ${ }^{75}$

Benítez elogió el trabajo de Marías a la vez que elucidó su envergadura:

Durante el período de la subvención, el profesor Marías trabajó intensamente y a fondo sobre la importancia de Ortega y Gasset como filósofo, escritor y maestro, así como en las contribuciones de Ortega a la vida cultural en España, Europa y América. Una vez que termine su trabajo será publicado en español en tres volúmenes bien documentados y extensos. El primero de estos, Ortega-Circunstancia y vocación [...] intenta interpretar el escenario cultural español de los siglos diecinueve y veinte, y la interacción de Ortega con ese escenario. He quedado profundamente impresionado por su extensión, cuidado y amenidad y

${ }^{74}$ RAC. RF. RG 1.2. Series 243. Caja 10. Carpeta 68. Julián Marías a John P. Harrison. Río Piedras, 8 de julio de 1960. Citado en español, en el original.

${ }^{75}$ RAC. RF. RG 1.1. Series 243. Caja 10. Carpeta 68. Carta de Jaime Benítez a John F. Harrison. Río Piedras, 19 de agosto de 1960. 
me complace notar que este primer volumen ya ha sido amplia y favorablemente reseñado tanto en España como América Latina. ${ }^{76}$

Quedaba la pregunta de si la Fundación renovaría la beca para Julián Marías, dado que había terminado el primero de sus libros sobre Ortega y Gasset y restaba al menos uno más por concluirse. De entrada, Benítez presentó a Harrison los resultados que Marías había alcanzado y luego los que éste anticipaba lograr.

El pasado julio discutí con Julián Marías sobre la orientación básica del segundo y tercer volúmenes. Él me mostró una muy detallada tabla de contenidos del segundo volumen. Marías trabaja en el borrador final. Contiene un análisis de las tesis y contribuciones principales de Ortega y Gasset e incluye una referencia directa a las circunstancias históricas e intelectuales inmediatas dentro de las cuales estas contribuciones fueron formuladas. Estoy convencido de que los tres volúmenes de Marías serán considerados como una contribución intelectual excepcional en sí mismos así como un tratado sobre Ortega de valor sumo a todos sus estudiantes, admiradores y críticos.

Julián Marías me comunica que se requerirá dedicarse por completo un año más para concluir su trabajo de manera exitosa. Está muy agradecido por la subvención que se le proporcionó y terminará su trabajo probablemente durante un período más largo de tiempo si no hay apoyo adicional viable. Creo, sin embargo, que deberíamos apoyarlo un año más y tomo la iniciativa en este asunto. La obra del señor Marías ha sido de lo más valiosa y estoy persuadido que los próximos dos volúmenes lo serán todavía más. ${ }^{77}$

En su reporte a Harrison, Benítez solicitó una subvención adicional de \$5,000 dólares que cubriera el período de septiembre de 1960 a septiembre de 1961, “como apoyo que le otorgaría la Universidad de Puerto Rico al profesor Julián Marías para culminar su trabajo actual

${ }^{76} \mathrm{Ibid}$. Las palabras en cursivas aparecen subrayadas en el original.

${ }^{77}$ RAC. RF. RG 1.1. Series 243. Caja 10. Carpeta 68. Carta de Jaime Benítez a John F. Harrison. Río Piedras, 19 de agosto de 1960. 
sobre José Ortega y Gasset”. Benítez le informó además a Harrison que le había pedido a Marías que permaneciera en la Universidad de Puerto Rico como asesor al rector con un salario anual 'sustancioso'. A Benítez le interesaba contratar también a Dolores Franco, esposa de Marías, quien era una "escritora distinguida por sus propios méritos". Le parecía a Benítez que ambos académicos serían de gran ayuda para la Universidad, dado que ésta tenía varios proyectos de libros de texto:

Todavía Marías podría trabajar en su libro sobre Ortega, aunque a un paso más tranquilo. El señor Marías agradeció mi invitación pero sintió que debería regresar a España para concentrarse en sus compromisos actuales. Aunque lo lamente, no puedo decir que desapruebo la decisión del señor Marías. El trabajo que hace es muy importante. Más aún, España no debería perder a las personas de capacidades excepcionales, buena voluntad y visión, que todavía luchan allí para preservar los valores intelectuales en los que creemos, a pesar de todas las tendencias en su contra. Pero hasta donde podamos deberíamos apoyarlos en su dedicación. ${ }^{78}$

Benítez cerró su reporte a Harrison con la esperanza que su solicitud fuera 'recibida favorablemente'. ${ }^{79}$ Harrison escribió de inmediato a Marías informándole de la carta de Benítez y solicitando una 'declaración breve' sobre “qué parte de su investigación y escritura total ha sido completada hasta ahora y su estimado de presupuesto en cuanto al apoyo financiero que usted necesitará para completar su trabajo" ${ }^{80}$

En lo que tocaba a Benítez y la posible subvención adicional de la Rockefeller, Marías indicó:

Estoy agradecido con Benítez y la Fundación, y siento que ustedes fueron sumamente generosos. Por supuesto que trabajaría sin preocu-

${ }^{78}$ Ibid.

${ }^{79}$ Ibid.

${ }^{80}$ RAC. RF. RG 1.2. Series. 243. Caja 10. Carpeta 68. Carta de John P. Harrison a Julián Marías. Nueva York, 25 de agosto de 1960. 
paciones económicas si tuviera una nueva subvención, pero estoy determinado a terminar mi trabajo como lo proyecté, tan pronto como sea posible de todas formas. [...] El segundo [volumen] está totalmente preparado, en detalle y parcialmente escrito. Espero terminarlo durante el próximo curso académico. [...] El último cubrirá los escritos publicados póstumamente y los escritos fragmentarios tras la Guerra Civil... Pienso que todo estará completado en 1962 (No me atrevo a ser más preciso: ars longa, como usted sabe). ${ }^{81}$

En breve, Marías aclaraba que su trabajo estaba encarrilado, y que se sentía comprometido a terminar su trabajo, con o sin apoyo financiero. Marías agregó: "nunca hubiera pensado en pedir una segunda subvención. El rector Benítez me dijo que sentía que no estaría injustificado. Sea cual fuere su decisión, trataré de continuar mi proyecto tan rápido y tan completamente como pueda, y estaré muy agradecido con la Fundación y con usted personalmente". ${ }^{82}$ Unas semanas más tarde, Marías reiteró que la Fundación había sido "extremadamente generosa y amable y no debería sentirse comprometida de manera alguna a extender la subvención". ${ }^{83}$

De esta manera Marías le proporcionó a Harrison una salida airosa, de la cual no tardó en valerse. En persona, Harrison le informó a Benítez que "no sería posible brindarle a la Universidad de Puerto Rico un apoyo posterior para este trabajo y que en adelante ésa era la postura de la RF". ${ }^{84}$

Una vez enterado de la postura de Harrison, Benítez insistió subrayando la capacidad intelectual de Marías y su postura favorable

${ }^{81}$ RAC. RF. RG 1.2. Series 243. Caja 10. Carpeta 68. Carta manuscrita de Julián Marías a John P. Harrison. Cambridge, 1 de septiembre 1960. Las palabras en cursivas aparecen subrayadas en el original.

${ }^{82}$ RAC. RF. RG 1.2. Series 243. Caja 10. Carpeta 68. Carta manuscrita de Julián Marías a John P. Harrison. Cambridge, 1 de septiembre 1960.

${ }^{83}$ RAC. RF. RG 1.2. Series 243. Caja 10. Carpeta 68. Carta manuscrita de Julián Marías a John P. Harrison. Madrid, 17 de octubre de 1960.

${ }^{84}$ RAC. RF. RG 1.2. Series 243. Caja 10. Carpeta 68. Entrevista de John P. Harrison con Julián Marías. Nueva York, 8 de septiembre de 1961. 
frente a los valores políticos estadounidenses: “el señor Marías es uno de los líderes más capaces y efectivos en el mundo español intelectual que vive en España hoy en día. Él es, además, uno de los muy pocos que tienen una comprensión inteligente y favorable de las posturas básicas y enfoques de Estados Unidos". ${ }^{85}$ Benítez añadió que había invitado de nuevo a Marías a trabajar de manera permanente en la Universidad de Puerto Rico pero que una vez más, dados sus compromisos en Madrid, había rechazado la invitación. ${ }^{86}$

Harrison tardó seis meses en retomar con Benítez el caso Marías. Cuando lo hizo, reinició el diálogo donde se había quedado: "Siento mucho que el señor Marías no ha sido capaz de aceptar su invitación para trabajar de manera permanente en Río Piedras", escribió Harrison: "aunque yo, como usted, sólo puedo aceptar su propio orden de prioridades". ${ }^{87}$ A la pregunta de si la Rockefeller ampliaría su subvención para permitirle a Marías terminar los dos volúmenes adicionales sobre Ortega y Gasset, Harrison explicó lo que sigue y con esto cerró el caso:

Desafortunadamente la naturaleza de nuestro programa actual imposibilita obtener una consideración favorable de apoyo adicional para la pesquisa del señor Marías. La subvención original fue excepcional para la Fundación en cuanto a que, de hecho, proveyó un subsidio al señor Marías para sus actividades intelectuales globales [sic] durante el período de 1956 a 1960. Pese a su propia importancia como una figura intelectual principal-y el todavía mayor interés del sistema filosófico de Ortega-, los compromisos en desarrollo de la Fundación en el presente son tales que ninguna excepción adicional a nuestro programa general puede obtener una consideración favorable. ${ }^{88}$

${ }^{85}$ RAC. RF. RG 1.2. Series 243. Caja 10. Carpeta 68. Jaime Benítez a John P. Harrison. Río Piedras, 13 de octubre de 1961.

${ }^{86}$ Ibid.

${ }^{87}$ RAC. RF. RG 1.2. Series: 243. Caja 10. Carpeta 68. John P. Harrison a Jaime Benítez. Nueva York, 4 de abril de 1962.

${ }^{88}$ Ibid. 
Así Harrison, como interlocutor de la Fundación, finiquitó este capítulo a la vez que dejó en claro que sabía que la Rockefeller no sólo patrocinó el proyecto de Marías otorgándole suficiente tiempo para lo redactara, sino todas sus actividades profesionales adicionales, que no cabían en el plan original de Marías.

\section{Epílogo}

Esta historia, para parafrasear al mismo Marías, tuvo otro colofón. Aunque Marías escribía con relativa rapidez (publicó por lo menos siete libros antes de cumplir los 37 años de edad, y cinco más entre 1952 y 1954), subestimó el tiempo que le tomaría completar su magnum opus sobre Ortega. Con la carga de hacer justicia a quien había sido su mentor intelectual, Marías cobró conciencia de que su tarea era mucho más ardua de lo que había anticipado. Para escribir 'un libro digno de Ortega', el autor reconstruyó la vida y pensamiento del filósofo así como la suya propia. Para Marías, el libro era un asunto personal (que debía limpiar el desaire también personal, cuando los hijos de Ortega y Gasset seleccionaron a otro filósofo para que editara los escritos inéditos e inacabados de su padre), que "requería revivir todos [sus] años desde [su] adolescencia, el proceso de absorción, comprensión, asimilación del pensamiento de Ortega como punto de partida para una filosofía que estaba en curso, en estado naciente", ${ }^{89}$

Una vez completado el primer volumen de su obra, confirmando así su compromiso de continuar, Marías comprendió que se había equivocado: "no se trataba de un libro en dos tomos, sino de una obra en dos libros". ${ }^{90}$ Como bien le escribió Marías a Harrison, darle coherencia y vigencia a los escritos de Ortega era adentrarse en un 'bosque tropical'. La metáfora era apta, pues evocaba recovecos oscuros, flora entretejida, y senderos no trillados en la obra de Ortega. El

${ }^{89}$ Julián Marías, Una vida presente, 2: 147.

${ }^{90} \mathrm{Ibid} ., 2$ : 148. Las palabras en cursivas aparecen así en el original. 
segundo libro, Las trayectorias, tardó 23 años en publicarse. Cuando Marías por fin lo concluyó, Jaime Benítez fue unos de sus mejores lectores. En palabras de Marías, "lo primero que hizo [Benítez], antes de escribirme, fue llamarme por teléfono desde San Juan, largamente, para comentar el nuevo libro, que ya no podía tener la primer lectura apasionada del primero". ${ }^{91}$

A lo largo de esas décadas, la amistad entre Marías y Benítez siguió afianzándose. Además de compartir una visión orteguiana de la vida, ambos eran ávidos lectores, locuaces interlocutores, amantes de la tertulia intelectual y de la poesía: los dos podían recitar de cabo a rabo Don Juan Tenorio. Marías continuó sus visitas anuales a Puerto Rico hasta el 1970, ofreciendo cursos cortos en mayo, mes que sus amigos bautizaron como el 'mes de Marías'. ${ }^{92}$ Marías colaboró además con Benítez para expandir y organizar la Universidad de Puerto Rico y, más particularmente al planificar el Colegio Universitario de Humacao. ${ }^{93}$ Cuando en 1972 Benítez ocupó el puesto de comisionado residente en Washington, D.C., Marías lo visitó en el Congreso estadounidense.

Aunque sus visitas a la isla se espaciaron y se tornaron más breves a partir de los años setenta, Marías siempre se sintió 'hondamente ligado a Puerto Rico'. ${ }^{94}$ No sorprende por tanto que, cuando Jaime Benítez murió en mayo de 2001, Marías publicara un tributo en el $A B C$ de Madrid, describiendo a Benítez como a su "amigo próximo, fraternal durante más de medio siglo", y destacando los vínculos que lo ataron a Puerto Rico, así como a Benítez. En una oración que revela los lazos entre ambos, el filósofo español subraya el éxito de Benítez ante las fundaciones norteamericanas, éxito que hizo posible una parte significativa de la obra de Marías: "Jaime Benítez gozaba de ilimitado prestigio en las grandes fundaciones intelectuales de los Estados Unidos, y he sido testigo de la respuesta inmediata que le han dado a

\footnotetext{
${ }^{91}$ Julián Marías, Una vida presente, 2: 154.

${ }^{92}$ Ibid., 2: 356.

${ }^{93}$ Ibid., 2:367.

${ }^{94}$ Ibid., 2: 127.
} 
la demanda de ayuda para acoger a grandes intelectuales en desgracia ante diversos poderes ilegítimos." 95 Marías no sólo fue testigo, sino uno de esos 'grandes intelectuales' que se beneficiaron del prestigio de Benítez y de la generosidad de la Fundación Rockefeller.

95 Julián Marías, "Un grande hispánico: Jaime Benítez”, $A B C$ (Madrid), 6 de junio de 2001. 